\title{
Monitoring molecular chirality exchange by photon echoes
}

\author{
František Šanda ${ }^{1, a}$ and Shaul Mukamel ${ }^{2}$ \\ 1 Charles University, Faculty of Mathematics and Physics, Ke Karlovu 5, Prague, 12116 Czech Re- \\ public \\ 2 Department of Chemistry, University of California, Irvine, CA 92697-2025
}

\begin{abstract}
We construct pulse polarization configurations in heterodyne four wave mixing for monitoring ultrafast (picosecond) exchange rates between optical isomers with axial chirality. This information is not available from linear circular dichroism, since enantiomers may not be isolated and racemate shows no chiral signal.
\end{abstract}

\section{Axial chirality in optical probes}

Despite the large differences between biological activity of optical isomers (enantiomers), all thermodynamic and most other physical properties are the same. Differences in optical response to circularly polarized light is a rare example of sensitive physical measurement. Two spectroscopic techniques are commonly used for detecting molecular chirality (right-handness): circular dichroism (CD) or Raman optical activity (ROA).

We consider molecular systems, whose chirality does not arise from organization of chemical bonds (e.g. asymmetric substitution of carbon atom) but is associated with chiral spacial arrangement of nonchiral units such as substituted biphenyles. Their planar structure is symmetric under reflection, but they can twist along dihedral angle $(\varphi)$ and assume chiral structure (see Fig 1). Such geometric rearrangement has a low barrier, so the chiral fluctuations and transitions can then occur on the picosecond timescale. It is hard to detect such ultrafast exchange between enantiomer pairs of chiral molecules. Unstable enantiomers may not be isolated, and racemic mixture shows no CD.

Monitoring of chiral transitions requires to detect CD signals along individual molecular trajectories. One obvious method is single molecule experiments. Taking CD or ROA to single molecule level is usually hard since chiral signals are three orders weaker then normal absorption. However, the detection of chiral optical response has been recently enhanced by constructing special confined electromagnetic fields, in which the CD signals are is enhanced to be comparable with absorption of linearly polarized light [1]. Another method is to look into molecular trajectories by nonlinear coherent spectroscopy [2].

We outline how nonlinear spectroscopy can be used to probe chiral exchange in a model molecule with dynamical axial chirality shown in Fig 1 . The planar molecule $\varphi=0$ is symmetric upon reflection, but can fluctuate toward chiral structures (e.g. the two enatiomers shown on left panel of Fig 1) controlled by the dihedral angle $\varphi \neq 0$.

Eigenstates of symmetric molecules can be sorted into symmetric $\Psi_{S}$ and antisymmetric $\Psi_{A}$ with respect to reflection. We assume that such classification remains meaningful also for finite $\varphi$. Electric $\mu$ and magnetic $M$ dipole moments are subject to symmetry rules, their components are even $(+1)$ or odd (-1) functions of dihedral angle. Let us define molecular frame, so that the symmetry plane is $y z$. General considerations show that the electric dipole of symmetric state $\mu_{S}$ and the magnetic dipole of antisymmetric state $M_{A}$ transform under reflection with signature $(-1,+1,+1)$, while $\mu_{A}$, $M_{S}$ has signature $(+1,-1,-1)$. To a good approximation, the molecular levels can be obtained as

\footnotetext{
a e-mail: sanda@karlov.mff.cuni.cz
} 

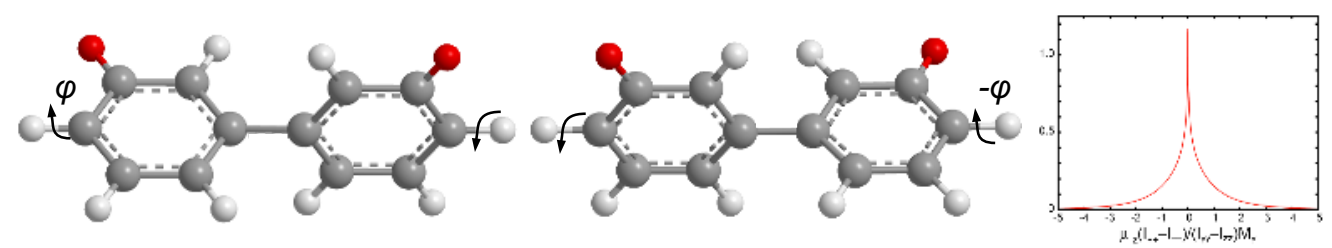

Fig. 1. Left: Optical isomers of substituted biphenyles. Axial chirality is controlled by dihedral angle $\varphi$. Right: Distributions of CD signal from single planar molecules.

symmetric (antisymmetric) linear combination of the wavefunction of the two phenyls and dipoles of chiral structures can be obtained from geometrical transformations of dipoles of planar structures [3]. Then, the electric dipoles are $\mu_{S}=\left(0,0, \tilde{\mu}_{S z} \cos \varphi\right), \mu_{A}=\left(\tilde{\mu}_{A x}, \tilde{\mu}_{S z} \sin \varphi, 0\right)$, magnetic dipoles $\mathbf{M}_{S}=\left(\tilde{M}_{S x}, \tilde{M}_{A z} \sin (\varphi),-\tilde{M}_{A y} \sin (\varphi)\right) \mathbf{M}_{A}=\left(0, \tilde{M}_{A y} \cos (\varphi), \tilde{M}_{A z} \cos (\varphi)\right)$. Here, $\tilde{\mu}_{S}, \tilde{M}_{S}\left(\tilde{\mu}_{A}, \tilde{M}_{A}\right)$ denote dipoles of symmetric $\Psi_{S}$ (antisymmetric $\Psi_{A}$ ) levels at planar configuration. Higher multipoles do not alter our key findings [3], we thus use the interaction Hamiltonian

$$
H_{\mathrm{m}-\mathrm{f}}=-\sum_{j} \mathbf{E}(t) \cdot\left[\mu_{j}-i \mathrm{k} \times \mathbf{M}_{j}\right] \Psi_{j}^{\dagger}+\text { h.c. }
$$

where $\mathrm{k}$ is direction (normalized wavevector) of plane-wave laser pulses of envelope $\mathbf{E}(t)$.

The CD signal is the difference between absorption of the right-handed $I_{++}$and left-handed $I_{--}$ circularly polarized light. CD signals from individual molecules vary with orientation in space [4]. In the lab frame, where light propagates along $x$ axis $(\mathrm{k}=(1,0,0))$, the CD signal is

$$
I_{++}-I_{--}=\operatorname{Im}\left(\mu_{y}^{L} M_{y}^{L}+\mu_{z}^{L} M_{z}^{L}\right)
$$

where $\mu^{L}, \mathbf{M}^{L}$ are lab frame dipoles. Distribution of CD signal of individual molecules with $\varphi=0$ along isotropic ensembles is shown in Fig 1c. Bulk measurements in gases or liquids provide ensemble averaging, where the connection between $\mathrm{CD}$ and molecular chirality is established $I_{++}-I_{--}=$ $(2 / 3) \operatorname{Im} \mu \cdot \mathbf{M} \sim-(1 / 3) \operatorname{Im} \tilde{\mu}_{S z} \tilde{M}_{A y} \sin (2 \varphi)$.

\section{Signatures of chirality exchange in coherent nonlinear spectrum}

Monitoring chirality exchange requires to correlate CD's (or other odd functions of $\varphi$ ) at two different times. This can be implemented by employing four-wave mixing. We have analyzed the photon-echo signal, in which the two correlation time intervals $t_{1}, t_{3}$ are short and the waiting time $t_{2}$ is used to observe molecular dynamic [2], and propose two pulse polarization configurations convenient to probe chirality exchange.

We first examine the following combination of pulse polarizations $\mathcal{R}_{++++}-\mathcal{R}_{++--}-\mathcal{R}_{--++}+\mathcal{R}_{----}$, which resembles the correlation of two CD's. This signal can be measured with various directions of incoming pulses, that can be used to eliminate variations of CD with orientation (shown in Fig 1). We found, that signals from parallel configuration (all four pulses propagates in $x$ direction, $\mathcal{R}^{x x}$ )) and perpendicular (e.g. first two pulses in $x$ direction, third pulse and local oscillator in $z$ direction, $\left.\mathcal{R}^{z x}\right)$ ) shall be combined to represent equally all three space directions. We next rewrite the indices of $\mathcal{R}$ from circular polarizations into terms of linear polarizations and taking into account $\mathcal{R}_{[y z][y z]}^{x x} \equiv$ $\mathcal{R}_{y z y z}^{x x}-\mathcal{R}_{z y y z}^{x x}-\mathcal{R}_{y z z y}^{x x}+\mathcal{R}_{z y z y}^{x x}=\mathcal{R}_{++++}^{x x}-\mathcal{R}_{++--}^{x x}-\mathcal{R}_{--++}^{x x}+\mathcal{R}_{----}^{x x}$, etc. we finally propose following signal

$$
\mathcal{R}_{c} \equiv \mathcal{R}_{[y z][y z]}^{x x}+\mathcal{R}_{[x y][y z]}^{y x}+\mathcal{R}_{[x y][y z]}^{z x}=\mathcal{R}_{[y z][y z]}^{x x}+2 \mathcal{R}_{[x y][y z]}^{z x}
$$

as a good candidate for measure of dynamical chirality. After orientation averaging (made along Ref. [5]) the signal $\mathcal{R}_{c}$ displays chirality exchange in particulary convenient form

$$
\mathcal{R}_{c}\left(0, t_{2}, 0\right)=\frac{16}{3}\left\langle\operatorname{Im}[\mu \cdot \mathbf{M}]\left(t_{2}\right) \operatorname{Im}[\mu \cdot \mathbf{M}](0)\right\rangle_{\varphi(t)} \approx \frac{4}{3}\left(\operatorname{Im} \tilde{\mu}_{S z} \tilde{M}_{A y}\right)^{2}\left\langle\sin 2 \varphi\left(t_{2}\right) \sin 2 \varphi(0)\right\rangle_{\varphi(t)} .
$$



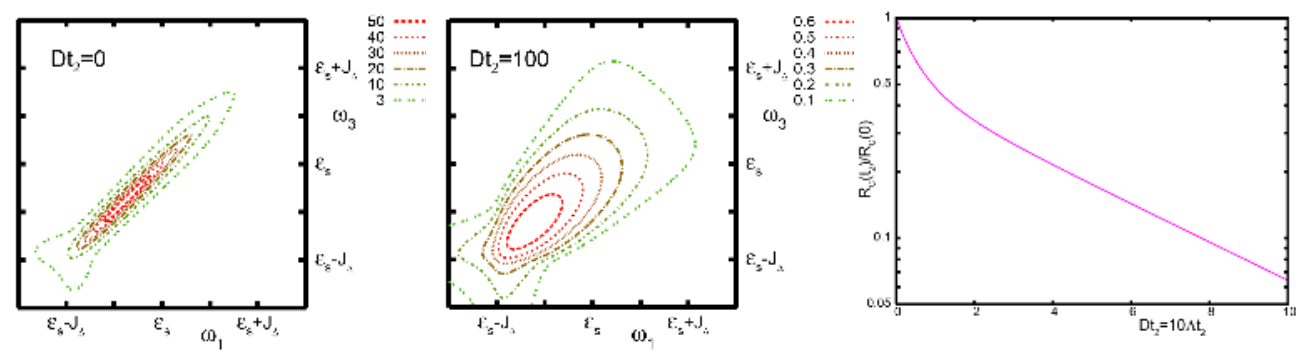

Fig. 2. Diffusion of $\varphi$ in double well potential has diffusion constant $D$ and transition rate (over barrier) $\Lambda=0.1 D$. Left:2D photon echo signal $\overline{\mathcal{R}}_{c}\left(\omega_{3}, t_{2}, \omega_{1}\right) \equiv \int_{0}^{\infty} \int_{0}^{\infty} e^{i\left(\omega_{1} t_{1}+\omega_{3} t_{3}\right)} \mathcal{R}_{c}\left(t_{3}, t_{2}, t_{1}\right) d t_{1} d t_{3}$ at two delay times $D t_{2}=0,100$. Right: Decay of $\mathcal{R}_{c}\left(0, t_{2}, 0\right)$ signal with delay time $t_{2}$.

Here, $\langle\ldots\rangle_{\varphi(t)}$ averages over ensemble of stochastic trajectories of dihedral angle [3]. Signal $\mathcal{R}_{c}$ provides similar information measured on both symmetric and antisymmetric states.

Fig 2., right panel shows $\mathcal{R}_{c}\left(t_{2}\right)$, when the dynamics of $\varphi(t)$ is modelled by diffusion in double well potential with minima at two enantiomer structures $\pm \varphi_{0}$. Two stage decay represent the equilibration inside well (short times) and transition over barrier (long times). We next analyzed the full 2D spectrum of photon echo signal. While the chirality exchange is primarily observed in peaks decay, the lineshapes evolution shows loss of correlation inside a well (Fig. 2, left).

A second proposed configuration employs the fact, that antisymmetric levels only acquire dipole moment in the chiral geometry. We found the following signal

$$
\mathcal{R}_{d}\left(t_{3}, t_{2}, t_{1}\right) \equiv \mathcal{R}_{y y z z}\left(t_{3}, t_{2}, t_{1}\right)-\mathcal{R}_{y z y z}\left(t_{3}, t_{2}, t_{1}\right)
$$

to carry signatures of chiral exchange quite similar to $R_{c}$.

$$
\mathcal{R}_{d}\left(0, t_{2}, 0\right)=\frac{1}{6}\left|\tilde{\mu}_{A x}\right|^{2} \tilde{\mu}_{S z}^{2}\left[\left\langle\sin ^{2} \varphi\left(t_{2}\right)\right\rangle_{\varphi}+\left\langle\sin ^{2} \varphi(0)\right\rangle_{\varphi}-2\left\langle\sin \varphi\left(t_{2}\right) \sin \varphi(0)\right\rangle_{\varphi}\right]
$$

The $\mathcal{R}_{d}$ signal which is not proportional to magnetic dipole, can be much stronger than $\mathcal{R}_{c}$. On the other hand its relation to dynamical chirality is not based on general geometric considerations, but is model specific. Signal (5) thus must be considered with some care [3].

Acknowledgement: Support of the National Institutes of Health (Grant No. GM59230), and the National Science Foundation (Grant No. CHE-0745892) and the Grant Agency of the Czech Republic (Grant No. 205/10/0989) is gratefully acknowledged. Mr. V. Perlík is acknowledged for technical help.

\section{References}

1. Y. Tang and A. E. Cohen, Science 332, (2011) 333.

2. S. Mukamel, Principles of Nonlinear Optical Spectroscopy (Oxford University Press, New York, 1995).

3. Šanda and S. Mukamel, J. Chem. Phys. 135, (2011) 194201.

4. F. Šanda, in preparation .

5. D. L. Andrews and T. Thirunamachandran, J. Chem. Phys. 67, (1977) 5026. 